Running Head: Language use and value orientations in multinational work teams

International Journal of Intercultural Relations, Volume 41, pp. 142-146, (c) 2014 by

\title{
Elsevier https://doi.org/10.1016/j.ijiintrel.2014.04.006
}

\section{Brief Report on}

Language use and value orientations in multinational work teams in Luxembourg: Conflict or harmony ${ }^{1}$

Jil Voss, Isabelle Albert \& Dieter Ferring University of Luxembourg

Autor's post-print

This article may not exactly replicate the final version published in International Journal of Intercultural Relations. It is not the version of record and is therefore not suitable for citation.

Please address correspondence to:

Dr. Isabelle Albert, Dipl.-Psych.

Isabelle.Albert@uni.lu

Research Unit INSIDE, Université du Luxembourg

Campus Walferdange - Bâtiment XII

Route de Diekirch

L-7220 Walferdange

${ }^{1}$ This study was part of the bachelor thesis of the first author at the University of Luxembourg. 
Running Head: Language use and value orientations in multinational work teams

\begin{abstract}
Due to globalization and international expansion of enterprises, cultural diversity in the workplace becomes more and more frequent. The present study focuses on two key issues regarding multinational work teams: language use and differences in value orientations of the employees of different cultural backgrounds and their potential effects on perception of team conflicts and team atmosphere. Results showed a relation between the perception of difficulties due to language use and the perception of conflicts in the team. Also, the difference between own and estimated value orientations of colleagues was positively related to the perception of conflicts and negatively to the evaluation of the team atmosphere.
\end{abstract}

Keywords: multinational work teams; value orientations; language use; conflict; team atmosphere; Luxembourg 
Running Head: Language use and value orientations in multinational work teams

\section{Introduction}

In a world of globalization, intercultural interaction becomes more and more frequent. In the workplace, people from different cultures encounter each other either in multinational teams or while interacting with companies located in different countries. On the one hand, this cultural diversity may provide synergy effects and increase competitiveness, as multiple cultural influences might enrich the perspectives of team members regarding work and life, for instance, by introducing new ideas or alternative work approaches to the team. On the other hand, organizations often also have to cope with distractions or conflicts due to cultural diversity in multinational teams (see Phillips \& Sackmann, 2002).

Regarding language use, members of multinational teams often speak mother tongues that differ from the language generally used at work. Differences in proficiency in the work language may constitute a barrier for communication as it can lead to misunderstandings or even harm coordination (e.g., Lauring \& Selmer, 2010). The communication style might also differ between team members depending on their cultural background, as some cultures prefer a more direct, others a more indirect communication style (e.g., Park et al., 2012), and this can constitute a further source of misinterpretations or conflicts. Last not least, language can be an important aspect of identification. Although multinational organizations often adopt a common official language in order to facilitate communication between their collaborators, further languages might be used in informal situations between coworkers (Lauring \& Selmer, 2010); consequently, employees might identify rather with group members of the same mother tongue in the sense of social categories of inclusion and exclusion (Giles \& Johnson, 1981).

Furthermore, team members from different cultural backgrounds might have different conceptions of what is good and desirable in line with their value orientations; they may thus differ in their preferences how to act and how they interpret and evaluate behavior of others (Hofstede, 2001; Schwartz, 2004). Whereas similar approaches to handle tasks can favor a 
Running Head: Language use and value orientations in multinational work teams

good team atmosphere, dissimilarities in the preferences and interpretation of behavior may negatively affect team effectiveness and performance if conflicts occur (see e.g., De Dreu \& Van Vianen, 2001). In line with this, Jehn and Mannix (2001) found in their longitudinal study that value consensus in the work group was related to lower levels of conflicts. They further suppose that value consensus among team members might create a positive group atmosphere in which different views about tasks can be openly discussed. Moreover, perceived similarities and differences in value orientations may again lead to ingroupoutgroup processes. According to social identity theory (Tajfel \& Turner, 1986), one might feel closer to those team members who are perceived to share the same value orientations (and vice versa), whereas one might distance from those who seem to cherish different values (see also Festinger, 1957). Also, the relation between attitude similarity and interpersonal attraction has been well established in social psychological research (e.g., Byrne, Griffitt, \& Stefaniak, 1967).

On the basis of these theoretical assumptions, the present study was designed to answer the questions if a) language use and b) value differences between coworkers have an impact on perceived conflicts and team atmosphere in an intercultural work environment in a multinational enterprise situated in the Grand Duchy of Luxembourg. Both criteria, perceived conflicts and team atmosphere, can be considered as crucial for organization development since they are both closely related to group performance and success of an organization (e.g., Souren and Sumati, 2009).

\section{Research questions and hypotheses}

With regard to language use, we focused on the subjective evaluation and comfort people feel with the generally used language both in team situations and during breaks. Whereas the effects of mere language proficiency have already been well studied in earlier research, the effects of subjective perceptions regarding language use have been rather 
Running Head: Language use and value orientations in multinational work teams

neglected so far (see Lauring and Selmer, 2010). We hypothesized that the more comfortable people are while speaking the generally used language in their firm, the fewer conflicts they will perceive in their team (Hypothesis 1) and the more positive they will evaluate the team atmosphere (Hypothesis 2). In particular, we were interested to know if a) the frequency of speaking a language other than the mother tongue at work, b) the employees' feeling that language is a source for problems in their team, c) their felt capacity to express any work related issue as well as d) their degree of comfort with the generally used language, is related to perceived conflicts and team atmosphere.

With regard to value orientations, we started from the assumption that not only the own value orientations per se might have an influence on how people behave (Hofstede, 2001; Schwartz, 2004), but- in line with social identity theory- the perception of the value orientations of colleagues in comparison to own value orientations may influence how people perceive conflicts or the team atmosphere (see also Jehn \& Mannix, 2001). It was hypothesized that the more people's own value orientations differ from the value orientations they attribute to their colleagues, the more conflicts are perceived (Hypothesis 3) and the larger the dissimilarity between the own values and the estimated values of the colleagues, the worse is the perceived team atmosphere (Hypothesis 4).

The present research questions are especially pertinent for the Luxembourg work context. Due to the small country size, companies in Luxembourg generally employ a high rate of cross-national collaborators such as commuters from the neighboring countries (i.e., Belgium, France, and Germany); furthermore, many international firms have subsidiaries in Luxembourg which attract collaborators from all over the world, and frequent international contacts are common. According to a recent study in Luxembourg, enterprises generally choose one language as common standard between coworkers, however, second and third languages are usually also used in most firms. Although $30 \%$ of the business leaders that participated in the survey (Berlitz Luxembourg, 2012) did not report any difficulties due to 
Running Head: Language use and value orientations in multinational work teams

multilingualism in their firms, a fourth of the respondents saw disadvantages due to misunderstandings as a consequence of a lack in language proficiency or due to a lack of intercultural understanding.

\section{Methods}

\section{Sample}

The sample of the present study was randomly chosen at a multinational company in Luxembourg with around 1,000 employees, where more than 40 nationalities are represented. The firm is part of a global network of professional firms providing Audit, Tax and Advisory services. The official company language is English.

For the present study, the human resources department at the participating firm sent the questionnaire to all the employees by e-mail. Participation was voluntary and confidential, anonymity was guaranteed. The time to fill out the form was about 15 minutes. The final sample comprised $N=87$ participants, $n=43$ male and $n=44$ female. They represented eleven nationalities with French (29.9\%), German (23\%), Luxembourgish (17.2\%), and Belgian (11.5\%) showing the highest percentages. Only 4 participants indicated a second nationality. Regarding the participants' mother tongues, $41.1 \%$ indicated French as the first language, $21.8 \%$ German and $16.1 \%$ Luxembourgish. A variety of further first languages were indicated such as English, Italian, Dutch, Portuguese, Russian, Filipino, Greek, Bengali, Bulgarian, and Chinese. Only five participants indicated a second mother tongue. Participants were aged between 22 and 48 years $(M=29.83, S D=5.65)$.

\section{Measures}

Participants filled out the standardized self-report questionnaire in English covering the following domains:

Language use. The generally used language at work was assessed both with regard to team situations as well as during breaks. Also, the participants' degree of comfort with the 
Running Head: Language use and value orientations in multinational work teams

language used in these two situations was evaluated on a 5-point Likert-scale $(1=$ "not comfortable at all" to 5 = "very comfortable"). Participants were further asked how often they speak a language that is not their mother tongue at work and if problems occurred in their team due to language, as well as if they are able to express any work related issue in the language generally used (from $1=$ "never" to 5 = "always").

Value orientations. To assess value orientations of the participants, a shortened version of the Schwartz Value Inventory (SVI) was used (Held, Müller, Deutsch, Grzechnik $\&$ Welzel, 2009; see also Schwartz, 2004). This questionnaire includes $k=10$ different value items describing prototypical persons, each assessing one value orientation represented in the Schwartz model (e.g., "It is important to this person to think up new ideas and be creative; to do things one's own way"), which had to be rated on a 6-point Likert-scale ( $1=$ "not at all like you" to 6 = "very much like you"). Further, participants were asked to indicate how they perceived their colleagues' value orientations by asking them to think about a typical member of their team and to indicate how much the described person is like this typical colleague. In order to assess perceived value (dis)similarity between respondents and their colleagues, we used a discrepancy score which was obtained by computing (for each participant) the mean of the differences (as absolute values) (d) between each of the ten ratings of actual own values (i.e. $\mathrm{X}_{\mathrm{act} i}$ ) and their corresponding estimation of a typical colleague's values (i.e. $\mathrm{X}_{\mathrm{est}}$ ) using the following formula (Scabini, Marta, \& Lanz, 2006; Roest et al., 2009):

$$
d=\frac{\sum_{i=1}^{k} \quad\left|\mathrm{X}_{\mathrm{act} i}-\mathrm{X}_{\mathrm{est} i}\right|}{k}
$$

Discrepancy scores obtained here ranged between 0.00 and 2.70 with $M=1.08(S D=0.49)$ where low values indicated a high and high values indicated a low perceived similarity between respondents and their colleagues. Reliability of the discrepancy score was satisfying ( $\alpha=.66)$. No differences between the main national groups were found $(F(3,65)=1.84$, n.s.). In order to check for differences between perceived value similarity and actual value 
Running Head: Language use and value orientations in multinational work teams

similarity between participants and their colleagues, we further computed a discrepancy score between respondents' actual own values and the mean rating of each of the ten values in the whole sample by using the same formula as mentioned above (where $\mathrm{X}_{\text {esti }}$ was substituted by $\left.M_{i}\right)$. The obtained scores of actual value discrepancy ranged between 0.39 and 1.78 with $M=$ $0.97(S D=0.30)$. Again, no differences between the main national groups were found $(F(3$, $67)=0.02$, n.s.). Interestingly, perceived value discrepancy was significantly higher than actual value discrepancy $(t(84)=2.02, p<.05)$.

Perceived conflicts were measured by a questionnaire by Jehn and Mannix (2001) that contains three dimensions of conflicts in work teams -"relationship conflict", "task conflict" and "performance conflict" with altogether $k=9$ items (e.g., "To what extent do you perceive relationship conflict in your team") rated on a 5-point Likert-scale from $1=$ "not at all" to $5=$ "quite a lot". Since the different dimensions correlated highly with each other (all over $r=.56$ $p<.01)$, a combined scale was used $(\alpha=.91, M=2.03, S D=0.69)$. No differences between main national groups were found $(F(3,67)=0.22$, n.s. $)$.

Team atmosphere was assessed with the four different dimensions "trust", "respect", "open discussion" and "cohesiveness" and altogether $k=10$ items (e.g., "To what extent you trust your fellow team members") to be rated on a 5-point Likert-scale ( $1=$ "not at all" to $5=$ “very much"; Souren \& Sumati, 2009, adapted from Jehn \& Mannix 2001). Again, since the correlations between the different dimensions were high (all over $r=.46, p<.01$ ), the combined scale was used $(\alpha=.89, M=3.80, S D=0.25)$. No differences between main national groups were found $(F(3,66)=1.99$, n.s. $)$.

\section{Results}

\section{Language use}

The language primarily used in a team situation was English as it was mentioned by $72 \%$ of the respondents; however, participants reported also using French (44\%), German (30\%) and 
Running Head: Language use and value orientations in multinational work teams

Luxembourgish $(8 \%)$ in team situations (it was possible to indicate more than one language). During breaks, French was the primarily used language as mentioned by $68 \%$ of the respondents, followed by English (46\%), German (36\%) and Luxembourgish (16\%). As a first analysis, we were interested in differences in main indicators between participants with a different mother tongue. We compared the largest represented groups of mother tongue, French ( $n=36$ participants), German $(n=19)$ and Luxembourgish $(n=14)$. Participants reported on average that they speak often a language that is not their mother tongue at work $(M=4.26, S D=0.96)$. There was no difference between the three main language groups $(F$ $(2,66)=1.49$, n.s. $)$. Problems due to language were rated as rather seldom on average $(M=$ $1.95, S D=0.88)$ and no differences between language groups were found here as well $(F(2$, $66)=0.34$, n.s.). Participants were in general also convinced that they are able to express any work related issue $(M=4.47, S D=0.66)$ without difference between the main language groups $(F(2,66)=1.28)$. Also, no significant difference was found between the main language groups regarding their comfort in language use at work $(F(2,66)=0.88$, n.s. $)$, whereas a tendency was found for language use during breaks $(F(2,66)=2.66, p<.10)$, where French native speakers felt most comfortable and Luxembourgish native speakers least comfortable. Comfort with language use in both situations was, however, generally rated as high $(M=4.46, S D=0.66 ; M=4.63, S D=0.63)$. The comfort with the language generally used during a team situation at work was significantly, but not very strongly, correlated with the comfort during a break situation $(r(87)=.27, p<.05)$.

Results in table 1 show that the hypotheses concerning language use and levels of conflicts (H1) as well as team atmosphere (H2) were mostly not supported by the data. An interesting result was however that the perception of the level of conflicts (but not the perceived team atmosphere) was related to the perception that problems in the team occur due to language.

-insert table 1- 
Running Head: Language use and value orientations in multinational work teams

\section{Value orientations}

In a first step of analysis, the largest national groups, French $(n=26)$, Belgian $(n=$ 10), German $(n=20)$ and Luxembourgish $(n=15)$ were compared with respect to their ratings of the ten value orientations. No significant differences were found ${ }^{2}$.

Nonetheless, the data supported hypotheses 3 and 4 (see table 1). Firstly, the larger the difference score between own values and estimated values of the colleagues, the more conflicts were perceived. Secondly, the larger the differences between own and estimated colleagues' values, the worse was the perceived team atmosphere.

\section{Summary and discussion}

In the present study, the essential question was about what influences the perception of conflicts and perceived team atmosphere in an intercultural work environment. Two aspects that were supposed to play a crucial role in multinational teams were focused on, a) language use and b) differences in perceived value orientations.

Although there was no relation between the comfort in language use and the perception of conflicts, the latter was positively related to the perception that problems in the team occur due to language. Since there was also no relation between frequency of use of a language other than mother tongue or felt own ability to express any work related issue and the perception of conflict, one may conclude that participants might "blame" the colleagues" rather than their own language capabilities or deficits for difficulties. In order to further explore this interpretation, we had a look at an additional open question about the sources for issues in the team that was part of the questionnaire. A total of 28 from 87 participants gave examples of issues, several indicating that there are communication and language problems (e.g., "pronouncing the words correctly", "linguistic aspects and misinterpretation of the

\footnotetext{
${ }^{2}$ Only two tendencies were found regarding the values "stimulation" and "conformity" with $F(3,67)=2.21$, and $F(3,67)=2.70, p<.10$, both being rated highest by French and lowest by German participants.
} 
Running Head: Language use and value orientations in multinational work teams

language", "misunderstandings regarding the tasks" and "different interpretations of words"). Future studies might use qualitative in-depth interviews to further explore this assumption.

There were no differences between the main national groups regarding preferences for value orientations. This is not that surprising, since the groups analyzed are all Western European cultures, sharing a similar historical and social background, which is supported by the Schwartz value map (Schwartz, 2004). More importantly, and irrespective of nationality, the perception of one's own in comparison to the colleagues' values was most strongly related to the perception of conflicts and team atmosphere. The results showed that the more different participants rated themselves compared to their colleagues in terms of value orientations, the more conflicts did they perceive and the worse was the reported team atmosphere. In line with social identity theory (Tajfel \& Turner, 1986), coworkers in multinational teams might rather identify with colleagues who are perceived to share similar values. Value similarity may however rather be a matter of subjective evaluation instead of objective differences between national groups or stereotypes about these groups. This interpretation was supported also by the fact that perceived value differences between participants and their colleagues were higher compared to the actual value differences between participants and the average ratings of the sample on the respective values. Participants might perceive colleagues whom they feel close to as sharing similar values in order to avoid cognitive dissonance (Festinger, 1957).

That perceived value differences can be an issue in multinational teams was again supported by the further qualitative information. Individual responses showed here that "cultural differences" and "what individuals consider normal, which comes from our education and social background" were reported as sources for issues in teams.

Limitations of the present study refer certainly to the subjectivity of the data as the actual level of language proficiency was not assessed. Also, selectivity of the sample could be an issue: it is possible that in particular coworkers with good language skills or with a special interest in the topic - for instance due to the own experience of conflicts in their team - 
Running Head: Language use and value orientations in multinational work teams

participated in the study. Certainly, it should be kept in mind that the present sample cannot be viewed as representative as only a small part of the coworkers of the contacted enterprise actually participated; nonetheless, as participation to the study was voluntary, it might be assumed that those who participated filled out the questionnaire faithfully. It provides therefore a good starting point for the study of intercultural communication in a typical work context in Luxembourg as it raises potential issues for conflicts and problems within multicultural teams.

The findings do certainly not indicate that there should be a complete value consensus between coworkers as some studies have even indicated that a certain amount of differences might also enhance team performance but they underline the potential of shared values. Future studies should also include direct indicators of team effectiveness in order to test how it might actually be affected by conflicts or differing values in the team.

In sum, multinational enterprises should reflect on shared values as a potential to enhance team performance by favoring mutual understanding and a positive team atmosphere. 
Running Head: Language use and value orientations in multinational work teams

\section{References}

Berlitz Luxembourg (2012). Multilingualism: Driver of competitiveness? Available at: http://www.berlitz.lu/upload/pdfs/WHITE_PAPER_MULTILINGUALISM.PDF

Byrne, D., Griffitt, W., \& Stefaniak, D. (1967). Attraction and similarity of personality characteristics. Journal of Personality and Social Psychology, 5, 82-90.

De Dreu, C. K. W., \& Van Vianen, A. E. M. (2001). Managing relationship conflict and the effectiveness of organizational teams. Journal of Organizational Behavior, 22, 309328.

Festinger, L. (1957). A theory of cognitive dissonance. Evanston, IL: Row \& Peterson.

Giles, H., \& Johnson, P. (1981). The role of language in ethnic group relations. In J. C. Turner \& H. Giles (Eds.), Intergroup Behaviour (pp. 199-243). Oxford: Blackwell.

Held, M., Müller, J., Deutsch, F., Grzechnik, E., \& Welzel, C. (2009). Value structure and dimensions. Empirical evidence from the German World Values Survey. World Values Research, 2, 55-76.

Hofstede, G. (2001). Culture's consequences: Comparing values, behaviors, institutions, and organizations across nations (2nd ed.). Thousand Oaks, California: Sage Publications, Inc.

Jehn, K. A., \& Mannix, E. A. (2001). The dynamic nature of conflict: A longitudinal study of intragroup conflict and group performance. The Academy of Management Journal, 44, 238-251.

Lauring, J., \&Selmer, J. (2010).Multicultural organizations: Common language and group cohesiveness. International Journal of Cross Cultural Management, 10, 267-284.

Park, H. S., Levine, T. R., Weber, R., Lee, H. E., Terra, L.I., et al., (2012).Individual and cultural variations in direct communication style. International Journal of Intercultural Relations, 36, 179-187. 
Running Head: Language use and value orientations in multinational work teams

Phillips, M. E., \& Sackmann, S. A. (2002). Managing in an era of multiple cultures. The Graziadio Business Report, 5(4).Available at: ttp://gbr.pepperdine.edu/024/multicultural.html

Roest, A. M., Dubas, J. S., Gerris, J. R. M., \& Engels, R. C. M. E. (2009). Value similarities among fathers, mothers, and adolescents and the role of a cultural stereotype: Different measurement strategies reconsidered. Journal of Research on Adolescence, 19, 812833.

Scabini, E., Marta, E., \& Lanz, M. (2006). The transition to adulthood and family relations. An intergenerational perspective. New York: Psychology Press.

Schwartz, S. H. (2004). Mapping and interpreting cultural differences around the world. In H. Vinken, J. Soeters, \& P. Ester (Eds.), Comparing cultures: Dimensions of culture in a comparative perspective. Leiden, The Netherlands: Brill.

Souren, P., \& Sumati, R. (2009). Cultural diversity, perception of work atmosphere, and task conflict in collaboration technology supported global virtual teams: Findings from a laboratory experiment. Proceedings of the 42nd Hawaii International Conference on System Sciences - 2009.

Tajfel, H., \& Turner, J. C. (1986). The social identity theory of intergroup behavior. In W. G. Austin \& S. Worchel (Eds.), The social psychology of intergroup relations (pp. 7-24). Chicago: Nelson-Hall. 